\title{
A Family of Partially Ordered Sets with Small Balance Constant
}

\author{
Evan Chen \\ Department of Mathematics \\ Massachusetts Institute of Technology, U.S.A. \\ evanchen@mit.edu
}

Submitted: Sep 18, 2017; Accepted: Nov 21, 2018; Published: Nov 30, 2018

(C) The author. Released under the CC BY license (International 4.0).

\begin{abstract}
Given a finite poset $\mathcal{P}=(P, \leqslant)$ and two distinct elements $x$ and $y$, we let $\operatorname{pr}_{\mathcal{P}}(x \prec y)$ denote the fraction of linear extensions of $\mathcal{P}$ in which $x$ precedes $y$. The balance constant $\delta(\mathcal{P})$ of $\mathcal{P}$ is then defined by

$$
\delta(\mathcal{P})=\max _{x \neq y \in P} \min \left\{\operatorname{pr}_{\mathcal{P}}(x \prec y), \operatorname{pr}_{\mathcal{P}}(y \prec x)\right\} .
$$

The $1 / 3-2 / 3$ conjecture asserts that $\delta(\mathcal{P}) \geqslant \frac{1}{3}$ whenever $\mathcal{P}$ is not a chain, but except from certain trivial examples it is not known when equality occurs, or even if balance constants can approach $1 / 3$.

In this paper we make some progress on the conjecture by exhibiting a sequence of posets with balance constants approaching $\frac{1}{32}(93-\sqrt{6697}) \approx 0.3488999$, answering a question of Brightwell. These provide smaller balance constants than any other known nontrivial family.
\end{abstract}

Mathematics Subject Classifications: 06A07, 05A14

\section{Introduction}

\subsection{Definitions}

Given a finite poset (partially ordered set) $\mathcal{P}=(P, \leqslant)$, and distinct elements $x, y \in P$, we let $\operatorname{pr}_{\mathcal{P}}(x \prec y)$ denote the proportion of linear extensions of $\mathcal{P}$ in which $x$ precedes $y$. In particular, $\operatorname{pr}_{\mathcal{P}}(x \prec y)+\operatorname{pr}_{\mathcal{P}}(y \prec x)=1$, and if $x \leqslant y$ in $\mathcal{P}$ then $\operatorname{pr}_{\mathcal{P}}(x \prec y)=1$.

The balance constant $\delta(\mathcal{P})$ is then defined by

$$
\delta(\mathcal{P})=\max _{x \neq y \in P} \min \left\{\operatorname{pr}_{\mathcal{P}}(x \prec y), \operatorname{pr}_{\mathcal{P}}(y \prec x)\right\} .
$$

(If $\mathcal{P}$ consists of one element, we let $\delta(\mathcal{P})=0$.) Thus $\delta(\mathcal{P}) \in\left[0, \frac{1}{2}\right]$ for any finite poset $\mathcal{P}$; in fact $\delta(\mathcal{P})=0$ exactly when $\mathcal{P}$ is a chain. 


\subsection{The $1 / 3-2 / 3$ Conjecture}

The main conjecture about balance constants is the famous $1 / 3-2 / 3$ conjecture.

Conjecture 1 (The $1 / 3-2 / 3$ conjecture). If $\mathcal{P}$ is a finite poset which is not a chain, then $\delta(\mathcal{P}) \geqslant \frac{1}{3}$.

This conjecture was first proposed in 1968 by Kislitsyn [9], then again by Fredman in 1976 [6] and Linial [10]. All three were motivated by the information-theoretic context of comparison sorting, but the problem is of course interesting in its own right.

The 1/3-2/3 conjecture has been studied extensively. The best bound which has been shown for all posets is due to Brightwell, Felsner, and Trotter [3] in 1995, who showed that

$$
\delta(\mathcal{P}) \geqslant \frac{5-\sqrt{5}}{10} \approx 0.276393
$$

whenever $\mathcal{P}$ is not a chain. This improved a result of Kahn and Saks [8] in 1984 which showed the weaker estimate $\delta(\mathcal{P}) \geqslant \frac{3}{11} \approx 0.272727$.

While still open for general partially ordered sets, the conjecture has been proven for several other families of partially ordered sets, for example:

- posets of width 2 (proved by Linial [10] in 1984)

- posets with a nontrivial automorphism (proved by Ganter, Hafuer, and Poguntke [7] in 1987),

- posets of height 2 (proved by Trotter, Gehrlein, Fishburn [17] in 1992),

- 5-thin posets (proved by Brightell and Wright [4] in 1992, though [13] implies it for 6 -thin posets, see below),

- semiorders (proved by Brightwell [5] in 1989),

- N-free posets (proved by Zaguia [18] in 2012).

- and posets whose Hasse diagram is a forest (proved by Zaguia [19] in 2016).

In 2006, Peczarski described an even stronger conjecture, the so-called "gold partition conjecture", which implies the 1/3-2/3 conjecture. Peczarski has proved the gold partition conjecture for several cases as well:

- posets with at most 11 elements [12] in 2006,

- 6 -thin posets [13] in 2008,

- posets with a nontrivial automorphism [14] in 2017,

- N-free posets [14] in 2017,

among others [14].

An extensive survey on the problem is given by Brightwell [2], which describes it as "one of the major open problems in the combinatorial theory of partial orders". 


\subsection{Posets with small balance constant}

The following example shows that the constant $1 / 3$ in best possible.

Example 2. Consider the poset $T$ with three elements $\{a, b, c\}$ with the single relation $a \leqslant b$ (shown in Figure 1). Then $\delta(T)=\frac{1}{3}$.

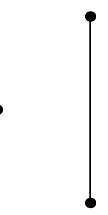

Figure 1: The poset $T$ with $\delta(T)=1 / 3$.

It follows that linear sums of $T$ and the singleton poset have balance constant $1 / 3$.

However, other than this example, little is known about the possible sets of balance constants. For example, it is not known whether there are any other posets which achieve a balance constant of exactly $1 / 3$, other than those in the example above. It is not even known whether balance constants can be arbitrarily close to $1 / 3$. On the other hand, for posets of width 2, Aigner [1] showed that posets which have width 2, and are not linear sums of copies of the singleton poset and $T$, must have balance constant strictly greater than $1 / 3$.

In Brightwell's survey [2, Section 4], an example of partially ordered set with $A$ with $\delta(A)=\frac{16}{45} \approx 0.355556$ is given. Brightwell also gives a family of partially ordered sets with balance constant approaching $\frac{7-\sqrt{17}}{8} \approx 0.359162$, and asks the following two questions.

Question 3. Is there a poset with balance constant between $\delta(T)=\frac{1}{3}$ and $\delta(A)=\frac{16}{45}$ ?

Question 4 . Is $\frac{7-\sqrt{17}}{8} \approx 0.359612$ the lowest possible limit point other than $1 / 3$ ?

Olson and Sagan [11] resolve the first question by finding a poset $C$ with

$$
\delta(C)=\frac{37}{106} \approx 0.34905660
$$

which at the time of submission of this paper, appeared to be the smallest balance constant exceeding $1 / 3$ in the literature. (See the next subsection for some improvements.) This poset is shown in Figure 2.

The aim of this paper is to answer both questions with a certain infinite family of partially ordered sets. We will prove the following theorem.

Theorem 5. There exists a sequence of posets whose balance constants approach

$$
\kappa=\frac{1}{32}(93-\sqrt{6697}) \approx 0.34889999 .
$$




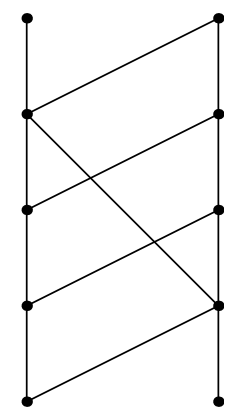

Figure 2: The poset $C$ from [11, Figure 13], with $\delta(C)=37 / 106$.

\subsection{Later results}

Subsequent to the submission of this paper, Peczarski [15] published numerical work which gives examples of partially ordered sets whose balance constants appear to approach a smaller value. In particular, there is an example of a poset $L$ such that

$$
\delta(L)=\frac{39148472052}{112223608848} \approx 0.3488434604 .
$$

This also answers the two questions supplied by Brightwell.

In private communications, Sah [16] has also constructed a family of posets whose balance constants approach

$$
\frac{5864893+27 \sqrt{57}}{16812976} \approx 0.34884346742240946 .
$$

Sah [16] has also proven that there exists an $\varepsilon>0$ such that $\delta(\mathcal{P})>\frac{1}{3}+\varepsilon$ for all posets $\mathcal{P}$ of width 2 with $\delta(\mathcal{P}) \neq \frac{1}{3}$, thus improving the result of [1].

\subsection{Roadmap}

The rest of the paper is divided as follows. In Section 2 we introduce the main players in our proof, and introduce the notation which we will need for the construction. Section 3 then provides explicit formulas for the number of linear extensions of our family of posets, and finally in Section 4 we compile these results together to prove the main theorem.

\section{Setup}

Definition 6. Throughout the paper let $\kappa=\frac{1}{32}(93-\sqrt{6697})$.

We first define a "master poset" from which our construction will derive.

Definition 7 . Let $\mathcal{P}_{\infty}$ denote the partially ordered set whose elements consist of two infinite $\mathbb{N}$-indexed chains

$$
\begin{gathered}
a_{1}<a_{2}<a_{3}<\cdots \\
b_{1}<b_{2}<b_{3}<\cdots
\end{gathered}
$$


together with the additional covering relations that

- $a_{i} \leqslant b_{i+1}$ whenever $i \equiv 1,2,3,4(\bmod 5)$, and

- $b_{j} \leqslant a_{j+2}$ whenever $j \equiv 0,2,4(\bmod 5)$.

All our constructions will be obtained by taking the bottom-most elements of either chain.

Definition 8. For positive integers $m$ and $n$ we let $\mathcal{P}(m, n)$ denote the sub-poset $\mathcal{P}_{\infty}$ induced by taking the elements $\left\{a_{1}, \ldots, a_{m}, b_{1}, \ldots, b_{n}\right\}$.

The example $\mathcal{P}(15,15)$ is shown in Figure 3.

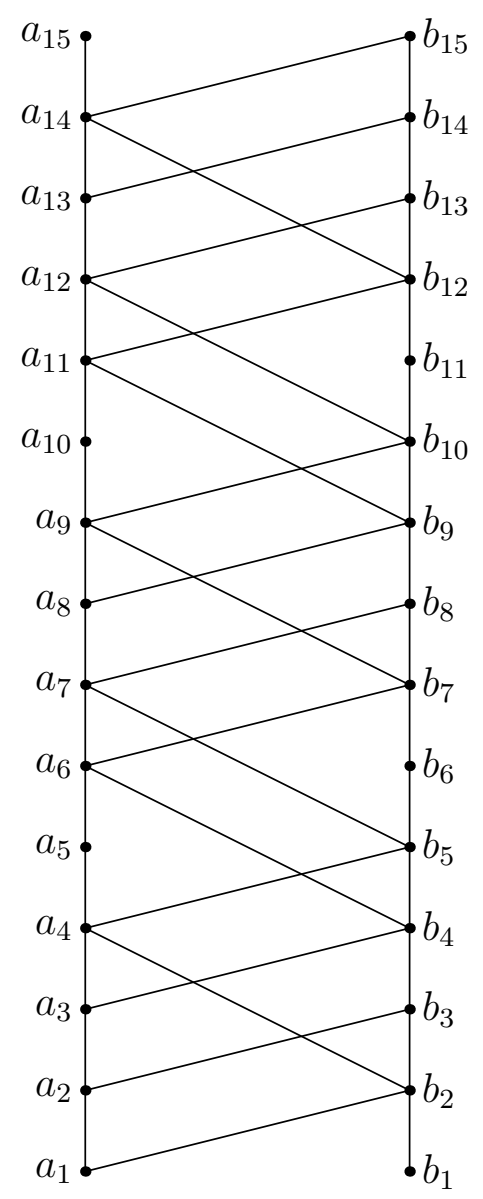

Figure 3: A picture of $\mathcal{P}(15,15)$.

Our main result is the following.

Theorem 9. As $k \rightarrow \infty$,

$$
\delta(\mathcal{P}(5 k, 5 k)) \rightarrow \kappa .
$$

To approach this result, we introduce further notation. 
Definition 10. Let $E(m, n)$ denote the number of linear extensions of $\mathcal{P}(m, n)$. For convenience we let $E(0, n)=E(m, 0)=1$ for positive integers $m$ and $n$, but we leave $E(0,0)$ undefined.

Then $E(m, n)$ may be computed recursively in the following way.

Proposition 11. For positive integers $m$ and $n$, we have

$$
E(m, n)= \begin{cases}E(m-1, n) & a_{m}>b_{n} \\ E(m, n-1) & a_{m}<b_{n} \\ E(m-1, n)+E(m, n-1) & \text { otherwise } .\end{cases}
$$

Proof. In a linear extension of $\mathcal{P}(m, n)$, either $a_{m}$ or $b_{n}$ must be the maximum element, and so the recursion follows by considering cases on this.

According to Proposition 11, the interesting cases are those for which $\mathcal{P}(m, n)$ has no maximum element. To this end, we introduce the following terminology.

Definition 12. We say the pair $(m, n)$ of positive integers is admissible if $\mathcal{P}(m, n)$ has no maximal element.

One can in fact characterize all the admissible pairs exactly. We obtain, essentially by definition, the following characterization.

Lemma 13. The pair $(m, n)$ is admissible if and only if it is one of the following forms:

1. $m=5 k+4$ and $n \in\{5 k+3,5 k+4\}$.

2. $m=5 k+3$ and $n \in\{5 k+1,5 k+2,5 k+3\}$.

3. $m=5 k+2$ and $n \in\{5 k+1,5 k+2\}$.

4. $m=5 k+1$ and $n \in\{5 k, 5 k+1\}$.

5. $m=5 k$ and $n \in\{5 k-2,5 k-1,5 k, 5 k+1\}$.

Remark 14. Note that this means that $(m, n)$ is admissible only if $|m-n| \leqslant 5$, in which case only the residues $m \bmod 5, n \bmod 5$ are relevant. In particular, if $(m, n)$ is admissible then so is $(m+5, n+5)$.

\section{Enumeration}

We now proceed to give explicitly compute $E(m, n)$ using induction. Several base cases are needed for this proof; we do not address these here, but simply record the results in Appendix A. In order to make this possible, we make the following observation.

Lemma 15. If $(m, n)$ is admissible then

$$
E(m+10, n+10)=164 E(m+5, n+5)-27 E(m, n) .
$$


Proof. When $\max (m, n) \leqslant 15$, the result follows by a manual inspection; see Appendix A for a table of values of $E(m, n)$. For the remaining cases, we proceed by induction on $m+n$. Assume $(m, n)$ is admissible and $\max (m, n)>15$. First assume $a_{m}$ and $b_{n}$ are not comparable, it follows that $a_{m-5}$ and $b_{n-5}$ are not comparable, and $a_{m-10}$ and $b_{n-10}$ are not comparable. Therefore, according to Proposition 11, we have

$$
\begin{aligned}
E(m, n) & =E(m-1, n)+E(m, n-1) \\
E(m-5, n-5) & =E(m-6, n-5)+E(m-5, n-6) \\
E(m-10, n-10) & =E(m-11, n-10)+E(m-10, n-11)
\end{aligned}
$$

By applying the inductive hypothesis, we have both

$$
\begin{aligned}
& E(m-1, n)=164 E(m-6, n-5)-27 E(m-11, n-10), \\
& E(m, n-1)=164 E(m-5, n-6)-27 E(m-10, n-11) .
\end{aligned}
$$

Thus it also follows that

$$
E(m, n)=164 E(m-5, n-5)-27 E(m-10, n-10)
$$

as desired.

The case where $a_{m}>b_{n}$ is done in the same fashion. This implies that $a_{m-5}>b_{n-5}$ and $a_{m-10}>b_{n-10}$, and so one applies Proposition 11 to conclude the same result, the only difference being that the terms $E(m, n-1), E(m-5, n-6)$ and $E(m-10, n-11)$ are omitted. One proceeds in exactly the same way for $a_{m}<b_{n}$.

This implies that the values of $E(m, n)$ satisfy a linear recurrence. Thus it makes sense to introduce the roots of the corresponding characteristic polynomial.

Definition 16. Throughout this paper, let

$$
\begin{aligned}
& \theta=82+\sqrt{6697} \approx 163.8352 \\
& \bar{\theta}=82-\sqrt{6697} \approx 0.1648
\end{aligned}
$$

be the two roots of the polynomial $t^{2}-164 t+27$.

Then, a direct computation using the results of Appendix A allows us to compute explicit closed forms:

Proposition 17. We have the following twelve closed forms.

$$
\begin{aligned}
& E(5 k+4,5 k+4)=\frac{3025}{2 \sqrt{6697}}\left(\theta^{k}-\bar{\theta}^{k}\right)+\frac{37}{2}\left(\theta^{k}+\bar{\theta}^{k}\right) \\
& E(5 k+4,5 k+3)=\frac{1883}{2 \sqrt{6697}}\left(\theta^{k}-\bar{\theta}^{k}\right)+\frac{23}{2}\left(\theta^{k}+\bar{\theta}^{k}\right) \\
& E(5 k+3,5 k+3)=\frac{571}{\sqrt{6697}}\left(\theta^{k}-\bar{\theta}^{k}\right)+7\left(\theta^{k}+\bar{\theta}^{k}\right)
\end{aligned}
$$




$$
\begin{aligned}
E(5 k+3,5 k+2) & =\frac{741}{2 \sqrt{6697}}\left(\theta^{k}-\bar{\theta}^{k}\right)+\frac{9}{2}\left(\theta^{k}+\bar{\theta}^{k}\right) \\
E(5 k+3,5 k+1) & =\frac{170}{\sqrt{6697}}\left(\theta^{k}-\bar{\theta}^{k}\right)+2\left(\theta^{k}+\bar{\theta}^{k}\right) \\
E(5 k+2,5 k+2) & =\frac{401}{2 \sqrt{6697}}\left(\theta^{k}-\bar{\theta}^{k}\right)+\frac{5}{2}\left(\theta^{k}+\bar{\theta}^{k}\right) \\
E(5 k+2,5 k+1) & =\frac{247}{2 \sqrt{6697}}\left(\theta^{k}-\bar{\theta}^{k}\right)+\frac{3}{2}\left(\theta^{k}+\bar{\theta}^{k}\right) \\
E(5 k+1,5 k+1) & =\frac{77}{\sqrt{6697}}\left(\theta^{k}-\bar{\theta}^{k}\right)+\left(\theta^{k}+\bar{\theta}^{k}\right) \\
E(5 k+1,5 k) & =\frac{93}{2 \sqrt{6697}}\left(\theta^{k}-\bar{\theta}^{k}\right)+\frac{1}{2}\left(\theta^{k}+\bar{\theta}^{k}\right) \\
E(5 k, 5 k+1) & =\frac{61}{2 \sqrt{6697}}\left(\theta^{k}-\bar{\theta}^{k}\right)+\frac{1}{2}\left(\theta^{k}+\bar{\theta}^{k}\right) \\
E(5 k, 5 k) & =\frac{77}{3 \sqrt{6697}}\left(\theta^{k}-\bar{\theta}^{k}\right)+\frac{1}{3}\left(\theta^{k}+\bar{\theta}^{k}\right) \\
E(5 k, 5 k-1) & =\frac{125}{6 \sqrt{6697}}\left(\theta^{k}-\bar{\theta}^{k}\right)+\frac{1}{6}\left(\theta^{k}+\bar{\theta}^{k}\right) \\
E(5 k, 5 k-2) & =\frac{16}{\sqrt{6697}}\left(\theta^{k}-\bar{\theta}^{k}\right) .
\end{aligned}
$$

\section{Computing the balance constant}

Throughout this section, we fix the poset $\mathcal{P}=\mathcal{P}(5 k, 5 k)$. With Proposition 17, we now turn to estimating the balance constant of $\mathcal{P}$. The point is that Proposition 17 essentially lets us compute $\operatorname{pr}_{\mathcal{P}}\left(a_{i} \prec b_{j}\right)$ for any $i$ and $j$ already. For example, we already have that

$$
\begin{aligned}
\operatorname{pr}_{\mathcal{P}}\left(a_{5 k} \prec b_{5 k}\right) & =\frac{E(5 k, 5 k-1)}{E(5 k, 5 k)} \\
& =\frac{\frac{1}{6}\left(\frac{125}{\sqrt{6697}}+1\right)}{\frac{1}{3}\left(\frac{77}{\sqrt{6697}}+1\right)} \quad \text { as } k \rightarrow \infty \\
& =\frac{1}{32}(\sqrt{6697}-61) \\
& =1-\kappa .
\end{aligned}
$$

Thus our goal is to show the following.

Proposition 18. For any $i, j \in\{1, \ldots, 5 k\}$ we have

$$
\min \left\{\operatorname{pr}_{\mathcal{P}}\left(a_{i} \prec b_{j}\right), \operatorname{pr}_{\mathcal{P}}\left(a_{j} \prec b_{i}\right)\right\} \leqslant \kappa .
$$


Proof. We give the full proof of Proposition 18 only in the case where $i \equiv 1(\bmod 5)$, since the other cases can be resolved in exactly the same fashion. For notational convenience, we set

$$
\begin{aligned}
& i=5 t+1 \\
& s=k-t .
\end{aligned}
$$

We will assume $t>0$, since the $t=1$ case corresponds to $\operatorname{pr}_{\mathcal{P}}\left(a_{1} \prec b_{1}\right)$ which is in any case equal to $\operatorname{pr}_{\mathcal{P}}\left(b_{5 n} \prec a_{5 n}\right)$ by symmetry.

Consider a linear extension $\prec$ of $\mathcal{P}$ then. Since $b_{5 t-1} \leqslant a_{5 t+1} \leqslant b_{5 t+2}$, we have three distinct possibilities.

\subsection{Case $b_{5 t-1} \prec a_{5 t+1} \prec b_{5 t}$}

Then if we add the relation $b_{5 t-1} \leqslant a_{5 t+1} \leqslant b_{5 t}$ to $\mathcal{P}$, the resulting poset is isomorphic to the linear sum of $\mathcal{P}(5 t, 5 t-1)$ and an inverted copy of $\mathcal{P}(5 s+1,5 s-1)$. An example with $(k, t)=(3,1)$ is shown in Figure 4 .

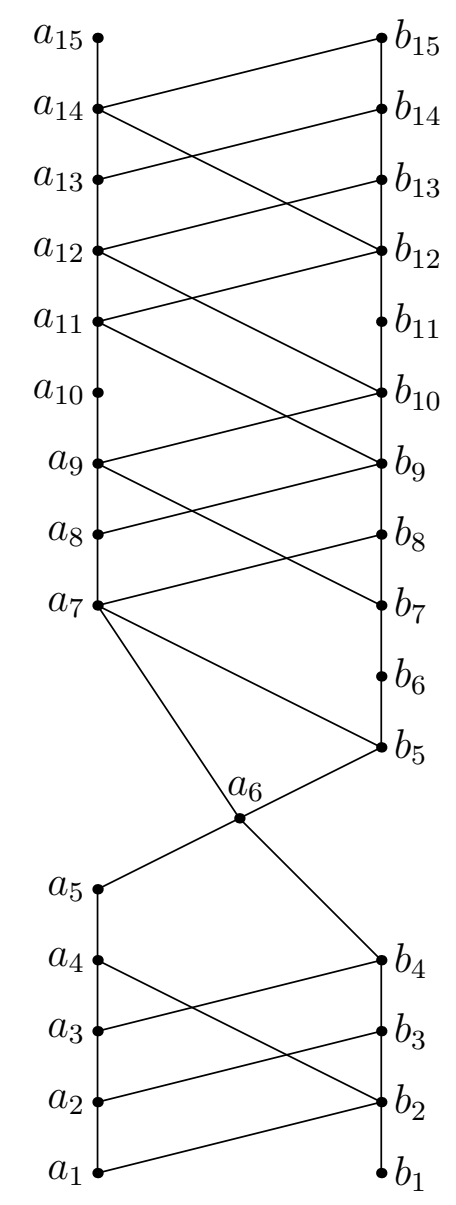

Figure 4: Adding the condition $b_{4} \leqslant a_{5} \leqslant b_{5}$ to $\mathcal{P}(15,15)$. 
The number of linear extensions in this case is then

$$
\begin{aligned}
E(5 t, 5 t-1) E(5 s+1,5 s-1) & =E(5 t, 5 t-1) E(5 s, 5 s-1) \\
& =\left[\frac{1}{6}\left(\frac{125}{\sqrt{6997}}+1\right)+o(1)\right]^{2} \theta^{k} \\
& =\left[\frac{11161+125 \sqrt{6697}}{12 \cdot 6697}+o(1)\right] \theta^{k} \\
& \approx(0.17745+o(1)) \theta^{k} .
\end{aligned}
$$

\subsection{Case $b_{5 t} \prec a_{5 t+1} \prec b_{5 t+1}$}

Then if we add the relation $b_{5 t} \leqslant a_{5 t+1} \leqslant b_{5 t+1}$ to $\mathcal{P}$, the resulting poset is isomorphic to the linear sum of $\mathcal{P}(5 t, 5 t)$ and an inverted copy of $\mathcal{P}(5 s, 5 s-1)$.

The number of linear extensions in this case is then $E(5 t, 5 t) E(5 s, 5 s-1)$, which equals

$$
\begin{aligned}
& E(5 t, 5 t) E(5 s, 5 s-1) \\
= & {\left[\frac{1}{3}\left(\frac{77}{\sqrt{6997}}+1\right)+o(1)\right]\left[\frac{1}{6}\left(\frac{125}{\sqrt{6997}}+1\right)+o(1)\right] \theta^{k} } \\
= & {\left[\frac{8161+101 \sqrt{6697}}{9 \cdot 6697}+o(1)\right] \theta^{k} } \\
\approx & (0.27253+o(1)) \theta^{k} .
\end{aligned}
$$

\subsection{Case $b_{5 t+1} \prec a_{5 t+1} \prec b_{5 t+2}$}

Then if we add the relation $b_{5 t} \leqslant a_{5 t+1} \leqslant b_{5 t+1}$ to $\mathcal{P}$, the resulting poset is isomorphic to the linear sum of $\mathcal{P}(5 t, 5 t+1)$ and an inverted copy of $\mathcal{P}(5 s-1,5 s-1)$.

Thus the number of linear extensions in this case is equal to

$$
\begin{aligned}
& E(4 t, 5 t+1) E(5 s-1,5 s-1) \\
= & {\left[\frac{1}{2}\left(\frac{61}{\sqrt{6997}}+1\right)+o(1)\right]\left[\frac{1}{2}\left(\frac{3025}{\sqrt{6997}}+37\right)+o(1)\right] \theta^{k-1} } \\
= & {\left[\frac{1411+15 \sqrt{6697}}{2 \cdot 6697}+o(1)\right] \theta^{k} } \\
\approx & {[0.19699+o(1)] \theta^{k} . }
\end{aligned}
$$

\subsection{Collating the cases}

On the other hand, the total number of linear extension of $\mathcal{P}$ is

$$
E(5 t, 5 t)=\left[\frac{1}{3}\left(\frac{77}{\sqrt{6697}}+1\right)+o(1)\right] \theta^{k}
$$




$$
\approx(0.64697+o(1)) \theta^{k}
$$

So, division gives

$$
\begin{aligned}
\operatorname{pr}_{\mathcal{P}}\left(b_{5 t-1} \prec a_{5 t+1} \prec b_{5 t}\right) & =\frac{1}{6}\left(-\frac{29}{\sqrt{6697}}+2\right)+o(1) \\
& \approx 0.27427+o(1) \\
\operatorname{pr}_{\mathcal{P}}\left(b_{5 t} \prec a_{5 t+1} \prec b_{5 t+1}\right) & =\frac{1}{6}\left(\frac{125}{\sqrt{6697}}+1\right)+o(1) \\
& \approx 0.42124+o(1) \\
\operatorname{pr}_{\mathcal{P}}\left(b_{5 t+1} \prec a_{5 t+1} \prec b_{5 t+2}\right) & =\frac{1}{2}\left(\frac{-32}{\sqrt{6697}}+1\right)+o(1) \\
& \approx 0.30449+o(1) .
\end{aligned}
$$

It follows that

$$
\min \left(\operatorname{pr}_{\mathcal{P}}\left(a_{5 t+1} \prec b_{j}\right), \operatorname{pr}_{\mathcal{P}}\left(b_{j} \prec a_{5 t+1}\right)\right)<\frac{1}{3}<\kappa
$$

for $j \in\{5 t, 5 t+1\}$, assuming $t>0$. Hence it holds for all $j$, since for $j \notin\{5 t, 5 t+1\}$ the left-hand side vanishes.

This completes the proof of Proposition 18 when $i \equiv 1(\bmod 5)$; the other four cases are analogous.

\section{Acknowledgments}

This research was funded by NSF grant 1659047, as part of the 2017 Duluth Research Experience for Undergraduates (REU). The author thanks Joe Gallian for supervising the research, and for suggesting the problem. The author is also grateful to Joe Gallian, and the anonymous referee, for comments on drafts of the paper.

\section{A Examples of values}

The following table lists the values of $E(m, n)$ for $\max (m, n) \leqslant 15$ (except for $E(0,0)$ undefined). The pairs $(m, n)$ which are admissible are bolded. 


\begin{tabular}{r|rrrrrrrrrrr}
$n=$ & 0 & 1 & 2 & 3 & 4 & 5 & 6 & 7 & 8 & 9 & 10 \\
\hline$m=0$ & & $\mathbf{1}$ & 1 & 1 & 1 & 1 & 1 & 1 & 1 & 1 & 1 \\
$m=1$ & $\mathbf{1}$ & $\mathbf{2}$ & 2 & 2 & 2 & 2 & 2 & 2 & 2 & 2 & 2 \\
$m=2$ & 1 & $\mathbf{3}$ & $\mathbf{5}$ & 5 & 5 & 5 & 5 & 5 & 5 & 5 & 5 \\
$m=3$ & 1 & $\mathbf{4}$ & $\mathbf{9}$ & $\mathbf{1 4}$ & 14 & 14 & 14 & 14 & 14 & 14 & 14 \\
$m=4$ & 1 & 4 & 9 & $\mathbf{2 3}$ & $\mathbf{3 7}$ & 37 & 37 & 37 & 37 & 37 & 37 \\
$m=5$ & 1 & 4 & 9 & $\mathbf{3 2}$ & $\mathbf{6 9}$ & $\mathbf{1 0 6}$ & $\mathbf{1 4 3}$ & 143 & 143 & 143 & 143 \\
$m=6$ & 1 & 4 & 9 & 32 & 69 & $\mathbf{1 7 5}$ & $\mathbf{3 1 8}$ & 318 & 318 & 318 & 318 \\
$m=7$ & 1 & 4 & 9 & 32 & 69 & 175 & $\mathbf{4 9 3}$ & $\mathbf{8 1 1}$ & 811 & 811 & 811 \\
$m=8$ & 1 & 4 & 9 & 32 & 69 & 175 & $\mathbf{6 6 8}$ & $\mathbf{1 4 7 9}$ & $\mathbf{2 2 9 0}$ & 2290 & 2290 \\
$m=9$ & 1 & 4 & 9 & 32 & 69 & 175 & 668 & 1479 & $\mathbf{3 7 6 9}$ & $\mathbf{6 0 5 9}$ & 6059 \\
$m=10$ & 1 & 4 & 9 & 32 & 69 & 175 & 668 & 1479 & $\mathbf{5 2 4 8}$ & $\mathbf{1 1 3 0 7}$ & $\mathbf{1 7 3 6 6}$ \\
$m=11$ & 1 & 4 & 9 & 32 & 69 & 175 & 668 & 1479 & 5248 & 11307 & $\mathbf{2 8 6 7 3}$ \\
$m=12$ & 1 & 4 & 9 & 32 & 69 & 175 & 668 & 1479 & 5248 & 11307 & 28673 \\
$m=13$ & 1 & 4 & 9 & 32 & 69 & 175 & 668 & 1479 & 5248 & 11307 & 28673 \\
$m=14$ & 1 & 4 & 9 & 32 & 69 & 175 & 668 & 1479 & 5248 & 11307 & 28673 \\
$m=15$ & 1 & 4 & 9 & 32 & 69 & 175 & 668 & 1479 & 5248 & 11307 & 28673
\end{tabular}

\begin{tabular}{r|rrrrr}
$n=$ & 11 & 12 & 13 & 14 & 15 \\
\hline$m=0$ & 1 & 1 & 1 & 1 & 1 \\
$m=1$ & 2 & 2 & 2 & 2 & 2 \\
$m=2$ & 5 & 5 & 5 & 5 & 5 \\
$m=3$ & 14 & 14 & 14 & 14 & 14 \\
$m=4$ & 37 & 37 & 37 & 37 & 37 \\
$m=5$ & 328 & 365 & 402 & 439 & 476 \\
$m=6$ & 318 & 318 & 318 & 318 & 318 \\
$m=7$ & 811 & 811 & 811 & 811 & 811 \\
$m=8$ & 2290 & 2290 & 2290 & 2290 & 2290 \\
$m=9$ & 6059 & 6059 & 6059 & 6059 & 6059 \\
$m=10$ & $\mathbf{2 3 4 2 5}$ & 29484 & 35543 & 41602 & 47661 \\
$m=11$ & $\mathbf{5 2 0 9 8}$ & 52098 & 52098 & 52098 & 52098 \\
$m=12$ & $\mathbf{8 0 7 7 1}$ & $\mathbf{1 3 2 8 6 9}$ & 132869 & 132869 & 132869 \\
$m=13$ & $\mathbf{1 0 9 4 4 4}$ & $\mathbf{2 4 2 3 1 3}$ & $\mathbf{3 7 5 1 8 2}$ & 375182 & 375182 \\
$m=14$ & 138117 & 242313 & $\mathbf{6 1 7 4 9 5}$ & $\mathbf{9 9 2 6 7 7}$ & 992677 \\
$m=15$ & 166790 & 242313 & 859808 & $\mathbf{1 8 5 2 4 8 5}$ & $\mathbf{2 8 4 5 1 6 2}$
\end{tabular}

\section{References}

[1] Martin Aigner. A note on merging. Order, 2(3):257-264, 1985.

[2] G. R. Brightwell. Balanced pairs in partial orders. Discrete Math., 201(1-3):25-52, 1999. 
[3] G. R. Brightwell, S. Felsner, and W. T. Trotter. Balancing pairs and the cross product conjecture. Order, 12(4):327-349, 1995.

[4] Graham Brightwell and Colin Wright. The 1/3-2/3 conjecture for 5 -thin posets. SIAM J. Discrete Math., 5(4):467-474, 1992.

[5] Graham R. Brightwell. Semiorders and the $\frac{1}{3}-\frac{2}{3}$ conjecture. Order, 5(4):369-380, 1989.

[6] Michael L. Fredman. How good is the information theory bound in sorting? Theoret. Comput. Sci., 1(4):355-361, 1975/76.

[7] Bernhard Ganter, Gerhart Häfner, and Werner Poguntke. On linear extensions of ordered sets with a symmetry. Discrete Math., 63(2-3):153-156, 1987. Special issue: ordered sets (Oberwolfach, 1985).

[8] Jeff Kahn and Michael Saks. Balancing poset extensions. Order, 1(2):113-126, 1984.

[9] S. S. Kislicyn. Finite partially ordered sets and their corresponding permutation sets. Mat. Zametki, 4:511-518, 1968.

[10] Nathan Linial. The information-theoretic bound is good for merging. SIAM J. Comput., 13(4):795-801, 1984.

[11] Emily J. Olson and Bruce E. Sagan. On the 1/3-2/3 conjecture. Order, Jan 2018.

[12] Marcin Peczarski. The Gold Partition Conjecture. Order, 23(1):89-95, 2006.

[13] Marcin Peczarski. The gold partition conjecture for 6-thin posets. Order, 25(2):91103, 2008.

[14] Marcin Peczarski. Comments on the golden partition conjecture. Contrib. Discrete Math., 12(1):106-109, 2017.

[15] Marcin Peczarski. The worst balanced partially ordered sets-ladders with broken rungs. Experimental Mathematics, 0(0):1-4, 2017.

[16] Ashwin Sah. Improving the $\frac{1}{3}-\frac{2}{3}$ conjecture for width two posets, 2018, arXiv: 1811.01500.

[17] W. T. Trotter, W. G. Gehrlein, and P. C. Fishburn. Balance theorems for height-2 posets. Order, 9(1):43-53, 1992.

[18] Imed Zaguia. The 1/3-2/3 conjecture for $N$-free ordered sets. Electron. J. Combin., 19(2):\#P29, 5, 2012.

[19] Imed Zaguia. The 1/3-2/3 conjecture for ordered sets whose cover graph is a forest, 2016, arXiv: $1610.00809 v 3$. 